\title{
Strange and heavy hadrons production from coalescence plus fragmentation in AA collisions at RHIC and LHC
}

\author{
Salvatore Plumari ${ }^{1,2, \star}$, Vincenzo Minissale ${ }^{1,2}$, Santosh K. Das ${ }^{1,2}$, Francesco Scardina ${ }^{1,2}$, and \\ Vincenzo Greco ${ }^{1,2}$ \\ ${ }^{1}$ Department of Physics and Astronomy, University of Catania, Via S. Sofia 64, 1-95125 Catania, Italy \\ ${ }^{2}$ Laboratori Nazionali del Sud, INFN-LNS, Via S. Sofia 62, I-95123 Catania, Italy
}

\begin{abstract}
In a coalescence plus fragmentation approach we study the $p_{T}$ spectra of charmed hadrons $D^{0}, D_{s}$ up to about $10 \mathrm{GeV}$ and the $\Lambda_{c}^{+} / D^{0}$ ratio from RHIC to LHC energies. In this study we have included the contribution from decays of heavy hadron resonances and also that due to fragmentation of heavy quarks that are left in the system after coalescence. The $p_{T}$ dependence of the heavy baryon/meson ratios is found to be sensitive to the heavy quark mass. In particular we found that the $\Lambda_{c} / D^{0}$ is much flatter than the one for light baryon/meson ratio like $p / \pi$ and $\Lambda / K$.
\end{abstract}

\section{Introduction}

Ultra-relativistic Heavy Ion Collisions (uHIC) can be used to probe the properties of the Quark-Gluon Plasma (QGP). In the studies of the QGP created in HIC is necessary to take into account that partonic behavior is not directly projected on the observables measured in UHIC. Thus the choice of the model for hadronization process is a crucial point in order to have a comparison with experimental data. The study of heavy baryons like $D$ mesons or $\Lambda_{c}$ baryons and the systematic study of the baryon over meson ratio for different species from light to heavy flavor are important in order to understand the hadronization mechanism. For light and strange hadrons it has been shown an enhancement of the baryon over meson ratio compared to the one for $\mathrm{p}-\mathrm{p}$ collision. Recent experimental results from STAR collaboration have shown that similar enhancement of the baryon/meson ratio is expected in the heavy flavor sector [1]. The coalescence hadronization process can explain both this features that appears at intermediate $p_{T}$. Hadronization via coalescence lead to a modification of the relative abundance of the different heavy hadron species produced. In particular this can manifests in a baryon-to-meson enhancement for charmed hadrons. The $\Lambda_{C} / D^{0}$ enhancement due to coalescence was first suggested in [2] where based on di-quark or three-quark coalescence mechanism with a full thermalized charm quarks and the predicted $\Lambda_{c} / D^{0}$ ratio is found to be comparable to the recent experimental data. Other recent calculations on the $\Lambda_{c} / D^{0}$ can be found in [3, 4].

\section{Hadronization via Coalescence and Fragmentation}

There are two hadronization mechanisms to produce heavy flavor hadrons: one is the fragmentation where high momentum quarks fragment directly in high momentum hadrons and another one is the

\footnotetext{
^e-mail: salvatore.plumari@hotmail.it
} 
coalescence where heavy quarks hadronize by recombination with light quarks. At increasing $p_{T}$ the probability to coalescence decreases and the standard fragmentation takes over.

\subsection{Fragmentation}

The hadron momentum spectra via fragmentation of minijet parton spectra is given by: $d N_{\text {had }} / d^{2} p_{T} d y=\sum_{j e t} \int d z \frac{d N_{j e t}}{d^{2} p_{T} d y} \frac{D_{\text {had } j \text { jet }\left(z, Q^{2}\right)}^{z^{2}}}{z^{2}}$ where $z=p_{\text {had }} / p_{\text {jet }}$ is the fraction of minijet momentum carried by the hadron and $Q^{2}=\left(p_{\text {had }} / 2 z\right)^{2}$ is the momentum scale for the fragmentation process. We employ the Peterson fragmentation function $D_{\text {had } / \text { jet }}\left(z, Q^{2}\right) \propto 1 /\left[z\left[1-\frac{1}{z}-\frac{\epsilon_{c}}{1-z}\right]^{2}\right][5]$. The $\epsilon_{c}$ is a free parameter fixed to $\epsilon_{c}=0.06$ by experimental data on $D$ mesons production in $p+p$ collisions [6]. In the absence of the $p+p$ data for the $\Lambda_{c}$ the value has been fixed to be a factor two larger than the $\mathrm{D}$ meson one [4]. For light quarks we employ the distribution function that at high $p_{T}>p_{0} \sim 2-3 \mathrm{GeV}$ is evaluated in next-to-leading order (NLO) in a pQCD scheme including the modification due to the jet quenching mechanism. The charm distribution function for both RHIC and LHC have been taken in accordance to the Fixed Order+Next-to-Leading Log (FONLL), as given in Ref.[7, 8].

\subsection{Coalescence}

The spectrum of hadrons formed by coalescence of quarks as developed in $[9,10]$ can be written as:

$$
\frac{d^{2} N_{H}}{d P_{T}^{2}}=g_{H} \int \prod_{i=1}^{n} \frac{d^{3} p_{i}}{(2 \pi)^{3} E_{i}} p_{i} \cdot d \sigma_{i} f_{q_{i}}\left(x_{i}, p_{i}\right) f_{H}\left(x_{1} \ldots x_{n}, p_{1} \ldots p_{n}\right) \delta^{(2)}\left(P_{T}-\sum_{i=1}^{n} p_{T, i}\right)
$$

where $d \sigma_{i}$ denotes an element of a space-like hypersurface, $g_{H}$ is the statistical factor to form a colorless hadron from quarks and antiquarks with spin $1 / 2$ while $f_{q_{i}}$ are the quark (anti-quark) phase-space distribution functions for i-th quark (anti-quark). For $n=2$ Eq. 1 describes meson formation while for $n=3$ the baryon one. For $D$ mesons the statistical factors is $g_{D}=1 / 36$ while for baryons i.e. $\Lambda_{c}$ the statistical factors is $g_{\Lambda}=1 / 108$. Finally $f_{H}\left(x_{1} \ldots x_{n}, p_{1} \ldots p_{n}\right)$ is the Wigner function and following Ref.[2, 10] we assume for $f_{H}$ a Gaussian shape in space and momentum relative coordinates $f_{H}\left(x_{1} \ldots x_{n}, p_{1} \ldots p_{n}\right)=A_{H} \exp \left(-\sum_{i=1}^{n-1} \frac{x_{r i}^{2}}{\sigma_{i}^{2}}-\sum_{i=1}^{n-1} p_{r i}^{2} \sigma_{i}^{2}\right)$ with $A_{H}$ a normalization constant for the Wigner function. While $\sigma_{i}$ are the covariant widths and are related to the size of the hadron and they can be evaluated from the charge radius of the hadrons according to quark model $[11,12]$. For $D$ meson the widths are fixed by the mean square charge radius of $D^{+}$which is given by $\left\langle r^{2}\right\rangle_{c h}=0.184 \mathrm{fm}^{2}$ and it correspond to $\sigma=0.283 \mathrm{GeV}$ while for $\Lambda_{c}^{+}$we have $\left\langle r^{2}\right\rangle_{c h}=0.15 \mathrm{fm}^{2}$ and the corresponding widths are $\sigma_{1}=0.18 \mathrm{GeV}$ and $\sigma_{2}=0.342 \mathrm{GeV}$. The overall normalization factor $A_{H}$ is fixed by requiring that the total recombination probability $P_{c o a l}=1$ for a $p=0$ heavy quark that undergoes to all possible heavy flavor hadron channels. From $P_{\text {coal }}$ we can assign a probability of fragmentation as $P_{\text {frag }}=1-P_{\text {coal }}$ and the charm distribution function undergoing to fragmentation is evaluated by convoluting the momentum of heavy quarks which do not undergone to coalescence. For the bulk we assume a thermalized system of gluons and $u, d, s$ quarks and anti-quarks where at $\tau=4.5 \mathrm{fm} / \mathrm{c}$ $(\tau=7.8 \mathrm{fm} / \mathrm{c})$ at RHIC (LHC) with a temperature of $T_{C}=165 \mathrm{MeV}$. The longitudinal momentum distribution is assumed to be boost-invariant in $y \in(-0.5,+0.5)$. For the quark-gluon plasma collective flow, we assume a radial flow profile as $\beta_{T}\left(r_{T}\right)=\beta_{\max } \frac{r_{T}}{R}$, where $R$ is the transverse radius of the fireball. For partons at low transverse momentum, $p_{T}<2 \mathrm{GeV}$ we consider a thermal distribution while $p_{T}>2 \mathrm{GeV}$, we consider the minijets that have undergone the jet quenching mechanism. The parametrization and the fireball parameters are the same used in [13]. For heavy quarks we use the transverse momentum distribution obtained by solving the relativistic Boltzmann equation that give a good description of $R_{A A}$ and $v_{2}$ of $D$ mesons [6]. 

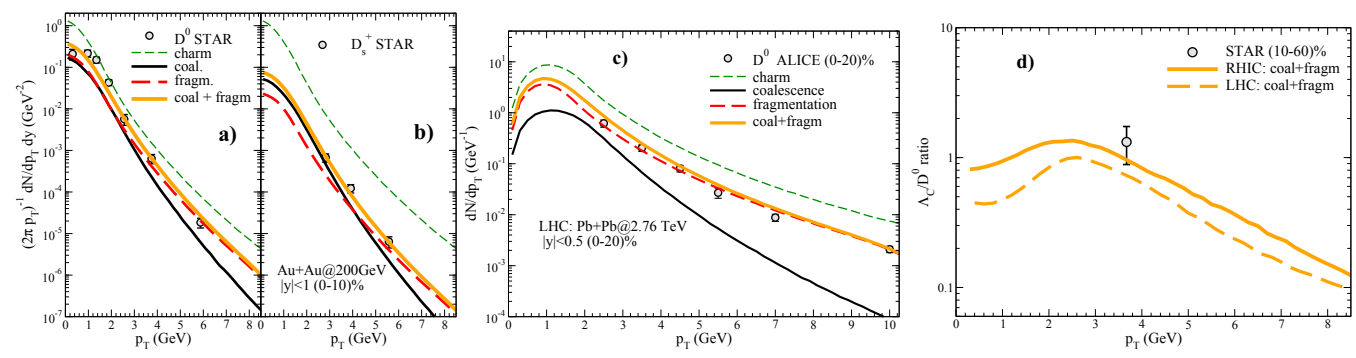

Figure 1. Left panel: $p_{T}$ spectra for $D^{0}$ and $D_{s}$ for $A u-A u$ collisions at $\sqrt{s}=200 \mathrm{GeV}$ and for $(0-10 \%)$ centrality. Green dashed line refers to the charm spectrum. Black solid and red dashed lines refer to the $D^{0}$ spectrum via only coalescence and only fragmentation respectively while orange solid line refers to the fragmentation plus coalescence processes. Data from [15]. Middle panel: $p_{T}$ spectra for $D^{0}$ for $P b-P b$ collisions at $\sqrt{s}=2.76 \mathrm{TeV}$ and for $(0-20 \%)$ centrality. Data from [16]. Right panel: $\Lambda_{c}^{+} / D^{0}$ ratio as a function of $p_{T}$. Solid line refer to $A u-A u$ collisions at $\sqrt{s}=200 \mathrm{GeV}$ while dashed line to $P b-P b$ collisions at $\sqrt{s}=2.76 \mathrm{TeV}$. Data from [1].

\section{Charmed hadrons transverse momentum spectra and ratio}

In this section, we show results for the transverse momentum spectra of $D^{0}$ and $D_{s}$ mesons and for $\Lambda_{c}$. In all the spectra the contribution that comes from resonances decay are included with their decay channel and the pertinent branching ratios as given in Particle Data Group [14]. For example for $D^{0}$ meson a dominant contribution comes from $D^{*+}$ and $D^{* 0}$ where $D^{*+} \rightarrow D^{0} \pi^{+}(68 \%)$ and $D^{* 0} \rightarrow D^{0} \pi^{0}$ $(62 \%)$ and $D^{* 0} \rightarrow D^{0} \gamma(38 \%)$. The inclusion of resonances improves the description at low $p_{T}$ but does not affect significantly the intermediate $p_{T}$. As shown in Fig.1 a) and b) the contribution of both hadronization mechanism (black solid and red dashed lines) is similar for $p_{T} \lesssim 2.5 \mathrm{GeV}$ while at higher $p_{T}$ the fragmentation becomes dominant mechanism. The contribution of coalescence plus fragmentation shown by orange lines give a good description of the experimental data. For the spectra of $D_{s}^{+}$we observe that at low $p_{T}$ Fig. $1 \mathrm{~b}$ ) the coalescence is the dominant mechanism while fragmentation play a role at $p_{T} \gtrsim 4 G e V$. This is related to the fact that the fragmentation fraction for $D_{s}^{+}$are small and less than about $8 \%$ of produced total heavy hadrons as obtained in [17]. Again the comparison with the experimental data shows that the description by both hadronization mechanisms appear to be quite good. In Fig. $1 \mathrm{c})$ it is shown the spectrum for $D^{0}$ meson for $(0-20 \%)$ centrality at LHC energies. As shown the total spectrum (coalescence plus fragmentation) shown by orange line is again in a good agreement with the experimental data. We notice that at LHC energies the fragmentation is the dominant hadronization mechanism to produce the $D^{0}$ meson. This is due to the fact that at high energies the coalescence is less significant because the effect of the coalescence depends on the slope of the charm quark momentum distribution. In fact for an harder charm quark distribution like at LHC the gain in momentum reflects in a smaller increase in the slope compared to the one at RHIC energies [6]. In Fig.1 d)it is shown the comparison between RHIC and LHC energies of the $\Lambda_{c}^{+}$to $D^{0}$ ratio as a function of $p_{T}$. As shown the coalescence by itself predicts a rise and fall of the baryon/meson ratio. The inclusion of fragmentation reduce the ratio and we can see that in the peak region we get a quite good agreement with the only experimental data. Notice that in our calculation we obtain similar baryon/meson ratio to the one predicted in [2]. Compared with measured light baryon/meson ratios like $\bar{p} / \pi^{-}$and $\Lambda / K_{S}^{0}$ ratios (see [18-20]), the obtained $\Lambda_{c}^{+} / D^{0}$ ratios has a different behavior it is thus much flatter and for $p_{T} \rightarrow 0$ hadronization by coalescence and fragmentation predict $\Lambda_{c}^{+} / D^{0} \simeq 0.8$ which is much larger respect to the one measured for light baryon/meson ratio with $\bar{p} / \pi^{-} \simeq 0$ for $p_{T} \rightarrow 0$ [13]. This behavior comes from the large mass of 
heavy quarks. Finally, we observe that at LHC energies coalescence plus fragmentation predict a smaller $\Lambda_{c}^{+} / D^{0}$. This is due to the fact that at LHC the hadronization by fragmentation becomes the dominant hadronization mechanism with the effect to reduce the baryon/meson ratio.

\section{Conclusions}

We have studied the transverse momentum spectra of charmed hadrons in heavy ion collisions from RHIC to LHC energies. The results obtained for the spectra are in good agreement with the recent experimental data from RHIC to LHC in central collisions. We have also studied the $p_{T}$ dependence of the $\Lambda_{c} / D^{0}$ ratio for different energies. The comparison with the light baryon/meson ratio shows that the $\Lambda_{c} / D^{0}$ ratio have a weaker dependence on the transverse momentum due to the massive charms quarks inside heavy hadrons. We have found that the $\Lambda_{c} / D^{0} \simeq 1.5$ at the peaks at $p_{T} \simeq 2.5 \mathrm{GeV}$ at RHIC energies and $\Lambda_{c} / D^{0} \simeq 0.8$ in the $p_{T} \simeq 0$ region. Similar results have been predicted in [2]. We observe that at LHC energies due to the fact that fragmentation start to be the dominant hadronization mechanism for the $D$ meson production this ratio is predicted to be smaller at higher energies.

\section{Acknowledgments}

V. Greco, S. Plumari acknowledge the support of the ERC-StG Grant under the QGPDyn project.

\section{References}

[1] L. Zhou (STAR) (2017), 1704.04364

[2] Y. Oh, C.M. Ko, S.H. Lee, S. Yasui, Phys. Rev. C79, 044905 (2009), 0901.1382

[3] S. Ghosh, S.K. Das, V. Greco, S. Sarkar, J.e. Alam, Phys. Rev. D90, 054018 (2014), 1407.5069

[4] S.K. Das, J.M. Torres-Rincon, L. Tolos, V. Minissale, F. Scardina, V. Greco, Phys. Rev. D94, 114039 (2016), 1604.05666

[5] C. Peterson, D. Schlatter, I. Schmitt, P.M. Zerwas, Phys. Rev. D27, 105 (1983)

[6] F. Scardina, S.K. Das, V. Minissale, S. Plumari, V. Greco (2017), 1707.05452

[7] M. Cacciari, P. Nason, R. Vogt, Phys. Rev. Lett. 95, 122001 (2005), hep-ph/0502203

[8] M. Cacciari, S. Frixione, N. Houdeau, M.L. Mangano, P. Nason, G. Ridolfi, JHEP 10, 137 (2012), 1205.6344

[9] V. Greco, C. Ko, P. Levai, Phys.Rev. C68, 034904 (2003), nucl-th/0305024

[10] V. Greco, C.M. Ko, R. Rapp, Phys. Lett. B595, 202 (2004), nucl-th/0312100

[11] C.W. Hwang, Eur. Phys. J. C23, 585 (2002), hep-ph/0112237

[12] C. Albertus, J.E. Amaro, E. Hernandez, J. Nieves, Nucl. Phys. A740, 333 (2004), nucl-th/0311100

[13] V. Minissale, F. Scardina, V. Greco, Phys. Rev. C92, 054904 (2015), 1502.06213

[14] K.A. Olive et al. (Particle Data Group), Chin. Phys. C38, 090001 (2014)

[15] L. Adamczyk et al. (STAR), Phys. Rev. Lett. 113, 142301 (2014), 1404.6185

[16] B. Abelev et al. (ALICE), JHEP 09, 112 (2012), 1203.2160

[17] M. Lisovyi, A. Verbytskyi, O. Zenaiev, Eur. Phys. J. C76, 397 (2016), 1509.01061

[18] S.S. Adler et al. (PHENIX), Phys. Rev. C69, 034909 (2004), nucl-ex/0307022

[19] S.S. Adler et al. (PHENIX), Phys. Rev. C71, 051902 (2005), nucl-ex/0408007

[20] B.B. Abelev et al. (ALICE), Phys. Lett. B736, 196 (2014), 1401.1250 